\title{
Clinicoradiological features of intraneural perineuriomas obviate the need for tissue diagnosis
}

\author{
${ }^{*}$ Thomas J. Wilson, MD, ${ }^{1}$ B. Matthew Howe, MD, ${ }^{2}$ Shelby A. Stewart, ${ }^{2}$ Robert J. Spinner, MD, ${ }^{3}$ and \\ Kimberly K. Amrami, MD²
}

1Department of Neurosurgery, Stanford University, Stanford, California; and Departments of ${ }^{2}$ Radiology and ${ }^{3}$ Neurosurgery, Mayo Clinic, Rochester, Minnesota

\begin{abstract}
OBJECTIVE This study aimed to define a set of clinicoradiological parameters with a high specificity for the diagnosis of intraneural perineurioma, obviating the need for operative tissue diagnosis.

METHODS The authors retrospectively reviewed MR images obtained in a large cohort of patients who underwent targeted fascicular biopsy and included only those patients for whom the biopsy yielded a diagnosis. Clinical and radiological findings were then tested for their ability to predict a tissue diagnosis of intraneural perineurioma. The authors propose a new set of diagnostic criteria, referred to as the Perineurioma Diagnostic Criteria. The sensitivity, specificity, positive predictive value, and negative predictive value of several clinicoradiological methods of diagnosis were compared.
\end{abstract}

RESULTS A total of 195 patients who underwent targeted fascicular biopsy were included in the cohort, of whom 51 had a tissue diagnosis of intraneural perineurioma. When the clinicoradiological methods used in this study were compared, the highest sensitivity $(0.86)$, negative predictive value $(0.95)$, and F1 score $(0.88)$ were observed for the decision trees generated in C5.0 and rPart, whereas the highest specificity (1.0) and positive predictive value (1.0) were observed for the Perineurioma Diagnostic Criteria.

CONCLUSIONS This study identified clinical and radiological features that are associated with a diagnosis of perineurioma. The Perineurioma Diagnostic Criteria were determined to be the following: 1) no cancer history, 2) unifocal disease, 3) moderate to severe hyperintensity on T2-weighted MR images, 4) moderate to severe contrast enhancement, 5) homogeneous contrast enhancement, 6) fusiform shape, 7) enlargement of the involved nerves, and 8) age $\leq 40$ years. Use of the Perineurioma Diagnostic Criteria obviates the need for tissue diagnosis when all of the criteria are satisfied. https://thejns.org/doi/abs/10.3171/2017.5.JNS17905

KEY WORDS perineurioma; fascicular biopsy; peripheral nerve

I NTRANEURAL perineurioma is a benign neoplasm derived from the perineurium of peripheral nerves. These tumors have a characteristic histopathological appearance with pseudo-onion bulb whorls of perineurial cells arranged around axons that may display varying degrees of degeneration. ${ }^{4,8} \mathrm{~A}$ major advance in the diagnosis of intraneural perineuriomas was the advent of immunohistochemical staining for epithelial membrane antigen (EMA). Intraneural perineuriomas are positive for EMA and negative for the Schwann cell marker S100, ${ }^{1,2}$ A variety of other names have been used in the literature when referring to this lesion, including localized hypertrophic neuropathy, intraneural neurofibroma, and pseudo-onion bulb neuropathy. ${ }^{2}$ Currently, the WHO terminology uses perineurioma. ${ }^{14}$

Intraneural perineurioma predominantly affects ado- lescents and young adults, with an equal predilection for males and females and the upper and lower extremities. ${ }^{9}$ Management of these lesions is controversial. ${ }^{3,6,7,9,11,13}$ Most favor targeted fascicular biopsy for tissue diagnosis. However, it is unclear if this is always necessary. The aim of the current study was first to determine the characteristic clinical and MRI features of intraneural perineuriomas based on a large cohort of patients who underwent targeted fascicular biopsy, and second, to determine if a clinicoradiological set of parameters alone is sufficient for diagnosis, obviating the need for targeted fascicular biopsy.

\section{Methods}

Study Design (Patient Cohort)

This study was approved by the institutional review

ABBREVIATIONS EMA = epithelial membrane antigen. SUBMITTED April 10, 2017. ACCEPTED May 23, 2017.

INCLUDE WHEN CITING Published online December 1, 2017; DOI: 10.3171/2017.5.JNS17905.

* Drs. Wilson and Howe contributed equally to this work. 
board of the Mayo Clinic. Due to the retrospective nature of this study, informed consent was not sought and was not required. Consecutive patients who underwent targeted fascicular biopsy at a single institution between November 2006 and April 2016 were identified. Their medical records, including pathology reports and radiological studies, were reviewed. Patients were excluded if they did not undergo MRI of the area of interest within 1 year preceding the biopsy. Patients were also excluded if the biopsy was nondiagnostic based on the pathology report. The medical records of the remaining patients underwent further review and analysis.

\section{Review of Imaging}

MRI studies for included patients were reviewed by 2 experienced musculoskeletal radiologists (B.M.H. and K.K.A.). For any of the analyzed features, when the radiologists disagreed, a neurosurgeon (T.J.W.) reviewed the study to adjudicate the disagreement. Both radiologists and the neurosurgeon reviewing the studies were blinded to diagnosis at the time of review. Prior to review of the MRI study, the radiologists were presented with a short patient vignette that included patient age, clinical presentation (pain, sensory, or motor symptoms), whether the patient's medical history included cancer (any form), and whether the disease process in question at the time of the imaging was thought to be unifocal or multifocal (terms defined below). The MRI features evaluated were the T1and T2-weighted signal characteristics, presence and pattern of postgadolinium enhancement (when gadolinium was administered), shape of the lesion, whether the involved nerve(s) was/were enlarged, presence of muscular fatty atrophy, and presence of subacute denervation.

The radiologists were also asked to grade the likelihood that the diagnosis was perineurioma: $5=$ definitely perineurioma (Fig. 1), 4 = perineurioma is the most likely diagnosis although there are other diagnostic considerations in the differential diagnosis, 3 = perineurioma is a possible diagnosis but at least 1 other diagnostic consideration in the differential diagnosis is equally as likely (Fig. 2), $2=$ perineurioma would be in the differential diagnosis but unlikely, and 1 = perineurioma would not be considered as part of the differential diagnosis (Fig. 3). This is referred to as the "perineurioma confidence scale."

\section{Review of Pathology}

No pathology specimens were reviewed as part of this study; pathological diagnoses were solely based on the available pathology reports in the medical record. When the diagnosis of hypertrophic neuropathy was given, the diagnosis was only considered positive for perineurioma if the pathologic description included those findings typical of perineurioma, including pseudo-onion bulbs that stained positively for EMA., 1,2,48

\section{Definition of Terms and Classification of Variables}

The clinical and demographic parameters collected included age at the time of MRI, sex, medical history of cancer (any form), clinical impression of the focality of disease (unifocal vs multifocal), and presenting clinical


FIG. 1. Perineurioma confidence scale 5: An 8-year-old boy with no history of cancer presented with painless weakness. A: Axial T2-weighted, fat-saturated MR images showing moderate hyperintensity in the right sciatic nerve (arrow) and a normal-appearing left sciatic nerve (arrowhead). B: Axial T1-weighted, fat-saturated, postgadolinium MR images showing avid, homogeneous contrast enhancement in the right sciatic nerve (arrow) and a normal-appearing left sciatic nerve without contrast enhancement (arrowhead). Biopsy confirmed intraneural perineurioma.

symptom. Unifocal disease was defined as involvement of a single, unilateral plexus, whereas multifocal disease was defined as involvement of $>1$ plexus. Clinical presentation was treated as a dichotomous categorical variable, dichotomized into pain at presentation or no pain at presentation.

T1-weighted appearance was treated as a categorical variable, with hypointense, isointense, and hyperintense defined as the signal intensity of the lesion relative to skeletal muscle. T2-weighted appearance was characterized as hypointense, isointense, or hyperintense. When the lesion was hyperintense to skeletal muscle, it was graded as follows: $1=$ mild, $2=$ moderate, and $3=$ severe. The variable was then dichotomized into none to mild hyperintensity and moderate to severe hyperintensity. Postgadolinium contrast enhancement was graded as follows: $0=$ nonenhancing, $1=$ mild, $2=$ moderate, and $3=$ severe (defined as the signal intensity of a slow-flowing vein). The variable was then dichotomized into none to mild contrast enhancement and moderate to severe contrast enhancement.

The pattern of enhancement was treated as a dichotomous categorical variable: homogeneous and heterogeneous. The shape of the lesion was treated as a dichotomous categorical variable: fusiform and nonfusiform. 



FIG. 2. Perineurioma confidence scale 3: A 65-year-old man with a history of lymphoma presented with pain. A: An axial T2-weighted, fat-saturated MR image showing severe hyperintensity in the left sciatic nerve (arrow). B: An axial T1-weighted, fatsaturated, postgadolinium MR image showing avid, homogeneous contrast enhancement in the left sciatic nerve (arrow). Biopsy confirmed lymphoma.

Enlargement of involved nerves was dichotomized into enlarged and normal. Muscular fatty atrophy was graded as follows: $0=$ none, $1=$ mild, $2=$ moderate, and $3=$ severe (defined as muscle atrophy with complete replacement of the muscle by fat). The variable was then dichotomized into none to mild and moderate to severe. Subacute denervation was treated as a dichotomous categorical variable: present and absent. Tissue diagnosis was treated as a dichotomous categorical outcome: perineurioma and other.

Through discussion with the reviewing radiologists about what features increased the likelihood of the diagnosis being a perineurioma, the Perineurioma Diagnostic Criteria were defined (prior to analysis). The Perineurioma Diagnostic Criteria included the following: 1) no cancer history, 2) unifocal disease, 3) moderate to severe hyperintensity on T2-weighted MRI, 4) moderate to severe contrast enhancement, 5) homogeneous contrast enhancement, 6) fusiform shape, 7) enlargement of the involved nerves, and 8) age $\leq 40$ years. Criteria were considered to have been met only if all 8 criteria were fulfilled.

\section{Outcome of Interest}

The outcome of interest was the ability to predict the tissue diagnosis of perineurioma on the basis of clinical and radiological criteria.

\section{Statistical Analysis}

Statistical analyses were performed using commercially available software (JMP version 10.0, SAS). Univariate comparison of continuous variables with a normal distribution was assessed using 2-sample t-tests, and continuous variables that did not meet the normality assumption were assessed using the Mann-Whitney U-test. All categorical data were assessed by chi-square test or Fisher's exact test, as appropriate.

Logistic regression was used to test univariate or multivariate associations between our variables of interest and the outcome of interest, which was diagnosis of perineurioma. We planned a priori to include in our multivariate analysis all of the variables associated with $p<0.05$ in the univariate analysis.

Methods that were tested for the ability to predict a diagnosis of perineurioma included the following: fulfilling all Perineurioma Diagnostic Criteria, fulfilling all Perineurioma Diagnostic Criteria minus those criteria concerning postcontrast imaging, fulfilling all criteria
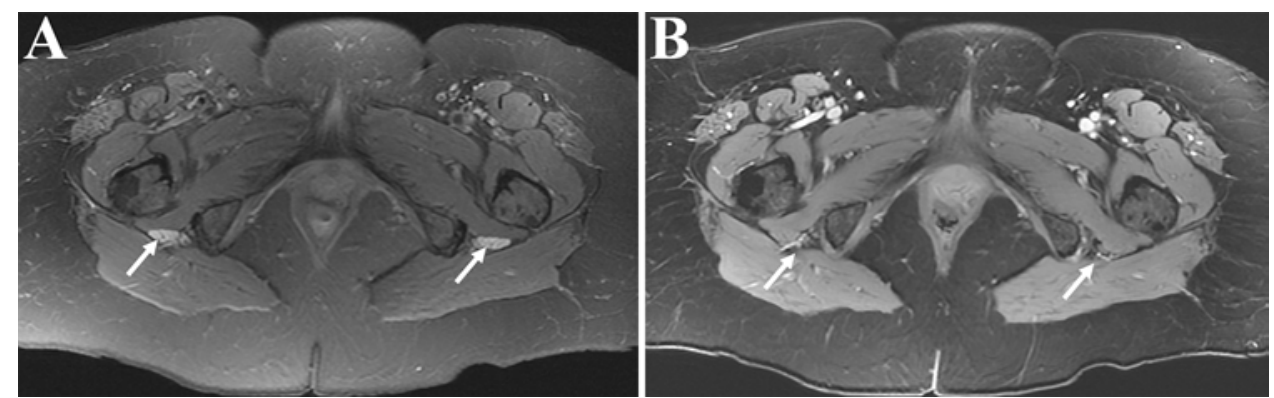

FIG. 3. Perineurioma confidence scale 1: A 61-year-old woman with no history of cancer presented with paresthesias. A: An axial T2-weighted, fat-saturated MR image showing severe hyperintensity in the enlarged bilateral sciatic nerves (arrows). B: An axial T1-weighted, fat-saturated, postgadolinium MR image showing no contrast enhancement in the bilateral sciatic nerves (arrows). Biopsy confirmed chronic inflammatory polyneuropathy. 
TABLE 1. Interrater reliability for the 2 radiologists assessing the imaging features

\begin{tabular}{|c|c|c|c|}
\hline Imaging Feature & $\begin{array}{c}\% \\
\text { Agreement }\end{array}$ & $\begin{array}{c}\kappa \\
\text { Coefficient }\end{array}$ & $\begin{array}{c}\kappa \\
\text { Coefficient } \\
\text { Range }\end{array}$ \\
\hline $\begin{array}{l}\text { Hyperintensity on T2-weighted } \\
\text { imaging }\end{array}$ & 91 & 0.61 & Substantial \\
\hline Contrast enhancement severity & 89 & 0.79 & Substantial \\
\hline $\begin{array}{l}\text { Contrast enhancement homo- } \\
\text { geneity }\end{array}$ & 91 & 0.81 & $\begin{array}{l}\text { Near } \\
\text { perfect }\end{array}$ \\
\hline Lesion shape & 92 & 0.64 & Substantial \\
\hline $\begin{array}{l}\text { Enlargement of involved } \\
\text { nerve(s) }\end{array}$ & 97 & 0.38 & Fair \\
\hline Fatty muscle atrophy & 86 & 0.72 & Substantial \\
\hline Subacute denervation changes & 90 & 0.79 & Substantial \\
\hline
\end{tabular}

found to be significant in multivariate logistic regression, the decision tree generated by the C5.0 statistical package in $\mathrm{R}$, and the decision tree generated by the rPart statistical package in R. For each method, the sensitivity [true positives/(true positives + false negatives)], specificity [true negatives/(true negatives + false positives)], positive predictive value [true positives/(true positives + false positives)], negative predictive value [true negatives/(true negatives + false negatives)], and F1 score $[2 \times\{$ (positive predictive value $\times$ sensitivity)/(positive predictive value + sensitivity)\}] were calculated.

Interrater reliability and agreement were assessed through percent agreement and Cohen's $\kappa$ coefficient. Percent agreement $>80 \%$ was considered to be high. Standard ranges for the $\kappa$ coefficient were used as follows: $<0=$ no agreement; $0-0.20=$ slight agreement; $0.21-0.40=$ fair agreement; $0.41-0.60=$ moderate agreement; $0.61-0.80=$ substantial agreement; and $0.81-1=$ near perfect agreement. ${ }^{5,10,15}$

A p value $<0.05$ was considered statistically significant for all analyses.

\section{C5.0 and rPart Statistical Packages in R}

Both the C5.0 and rPart statistical packages in R were used to generate decision trees for the diagnosis of perineurioma versus other. The C5.0 algorithm works on the principle of maximizing normalized information gain to select attributes that optimally divide the total population, in this case into perineurioma versus other. The algorithm uses a process of boosting and winnowing to optimize and improve the accuracy of the decision tree. rPart operates on similar principles, first selecting the variable that best splits the total data into 2 groups. This process is reiterated for each subgroup until no further improvement in the separation is seen. The algorithm then uses cross-validation to trim the tree.

\section{Results}

\section{Patient Cohort}

A total of 195 patients who underwent targeted fascicular biopsy were included in the cohort, 51 of whom had a
TABLE 2. Bivariate comparison of the cohort of patients diagnosed with a perineurioma versus another diagnosis

\begin{tabular}{|c|c|c|c|}
\hline Variable & $\begin{array}{c}\text { Other } \\
\text { Diagnosis }\end{array}$ & Perineurioma & $\begin{array}{c}p \\
\text { Value }\end{array}$ \\
\hline No. of patients & 144 & 51 & \\
\hline Mean age $\pm S D$, yrs & $55 \pm 16$ & $24 \pm 15$ & $<0.001$ \\
\hline Sex & & & 0.88 \\
\hline Male & $78(54)$ & $27(53)$ & \\
\hline Female & $66(46)$ & $24(47)$ & \\
\hline Pain at presentation & $50(35)$ & $4(8)$ & $<0.001$ \\
\hline Cancer history & $53(37)$ & $0(0)$ & $<0.001$ \\
\hline Unifocal disease & $88(61)$ & $48(94)$ & $<0.001$ \\
\hline $\begin{array}{l}\text { Moderate to severe hyperinten- } \\
\text { sity on T2-weighted imaging }\end{array}$ & $106(74)$ & $48(94)$ & $<0.01$ \\
\hline $\begin{array}{l}\text { Moderate to severe enhance- } \\
\text { ment }\end{array}$ & $41 / 136(30)$ & $41 / 42(98)$ & $<0.001$ \\
\hline Homogeneous enhancement & $25 / 136(18)$ & $39 / 42(93)$ & $<0.001$ \\
\hline Fusiform shape & $114(79)$ & $48(94)$ & 0.01 \\
\hline Enlargement of involved nerves & $137(95)$ & $51(100)$ & 0.11 \\
\hline Moderate to severe fatty atrophy & $72(50)$ & $30(59)$ & 0.28 \\
\hline Subacute denervation & $97(67)$ & $27(53)$ & 0.07 \\
\hline
\end{tabular}

Values are number (\%) of patients unless stated otherwise.

tissue diagnosis of perineurioma; the remaining 144 patients had another diagnosis (not perineurioma). The mean age $( \pm$ SD) at imaging for the cohort was $47( \pm 20)$ years, with a range of 3-86 years. The cohort had a slight male predominance (54\% male, $46 \%$ female). Postcontrast images were available for review for 178 patients (91\%).

\section{Interrater Reliability}

The agreement of the 2 radiologists was high based on percentage agreement for all imaging features reviewed (Table 1). The interrater reliability based on the $\kappa$ statistic was near perfect for assessment of the homogeneity of contrast enhancement; substantial for severity of hyperintensity on T2-weighted imaging, severity of contrast enhancement, shape of the lesion, fatty muscle atrophy, and subacute denervation changes; and fair for enlargement of the involved nerve(s).

\section{Bivariate Comparison of Perineurioma Versus Other}

Significant differences in clinical and radiological parameters were observed between the patients diagnosed with a perineurioma and those with another diagnosis (Table 2). The groups differed with regard to age at imaging, pain at clinical presentation, clinical history of cancer, focality of disease, hyperintensity on T2-weighted imaging, contrast enhancement severity and pattern, and shape of the lesion.

\section{Logistic Regression Analysis Predicting Perineurioma Diagnosis}

Univariate logistic regression analysis was performed to test the ability of the variables of interest to predict a tissue diagnosis of perineurioma (Table 3). Negative pre- 
TABLE 3. Univariate and multivariate logistic regression analysis of factors predictive of a diagnosis of perineurioma

\begin{tabular}{|c|c|c|c|c|}
\hline Variable & $\begin{array}{l}\text { Univariate Odds Ratio } \\
\qquad(95 \% \mathrm{Cl})\end{array}$ & $\begin{array}{l}\text { Univariate } \\
\text { p Value }\end{array}$ & $\begin{array}{l}\text { Multivariate Odds Ratio } \\
\qquad(95 \% \mathrm{Cl})\end{array}$ & $\begin{array}{l}\text { Multivariate } \\
\text { p Value }\end{array}$ \\
\hline Age & $0.90(0.87-0.93)$ & $<0.001$ & $0.91(0.85-0.96)$ & $<0.001$ \\
\hline Male sex & $0.95(0.50-1.81)$ & 0.88 & & \\
\hline Pain on presentation & $0.16(0.05-0.42)$ & $<0.001$ & $0.17(0.02-1.30)$ & 0.09 \\
\hline Cancer history & $0.03(0.002-0.16)$ & $<0.001$ & $0.09(0.004-0.75)$ & 0.02 \\
\hline Unifocal disease & $10.18(3.51-43.27)$ & $<0.001$ & $10.95(0.72-182.29)$ & 0.08 \\
\hline Moderate to severe hyperintensity on T2-weighted imaging & $5.74(1.95-24.55)$ & $<0.001$ & $0.94(0.11-8.95)$ & 0.96 \\
\hline Moderate to severe enhancement & $94.99(19.61-1712.09)$ & $<0.001$ & $99.48(6.07-5921.05)$ & $<0.001$ \\
\hline Homogeneous enhancement & $57.72(19.03-252.37)$ & $<0.001$ & $75.38(7.64-1743.62)$ & $<0.001$ \\
\hline Fusiform shape & $4.21(1.41-18.14)$ & $<0.01$ & $0.18(0.01-1.91)$ & 0.16 \\
\hline Enlargement of involved nerves & $2.55(0.44-48.37)$ & 0.33 & & \\
\hline Moderate to severe fatty atrophy & $1.43(0.75-2.75)$ & 0.28 & & \\
\hline Subacute denervation & $0.54(0.28-1.05)$ & 0.07 & & \\
\hline
\end{tabular}

dictive factors included increasing age, history of pain at presentation, and clinical history of cancer. Positive predictive factors included unifocal disease, moderate to severe hyperintensity on T2-weighted imaging, moderate to severe contrast enhancement, homogeneous contrast enhancement, and fusiform shape. Age as a continuous variable was a negative predictor (increasing age decreased the likelihood of a diagnosis of perineurioma). The most significant categorical variable reducing the likelihood of perineurioma was a history of cancer (OR $0.03,95 \%$ CI $0.002-0.16$ ), whereas the most significant categorical variable increasing the likelihood of perineurioma was moderate to severe contrast enhancement (OR 94.99, 95\% CI 19.61-1712.09).

Multivariate logistic regression analysis was then performed with the significant factors from the univariate logistic regression analyses (Table 3 ). Only age, clinical history of cancer, moderate to severe contrast enhancement, and homogeneous contrast enhancement remained signifi-

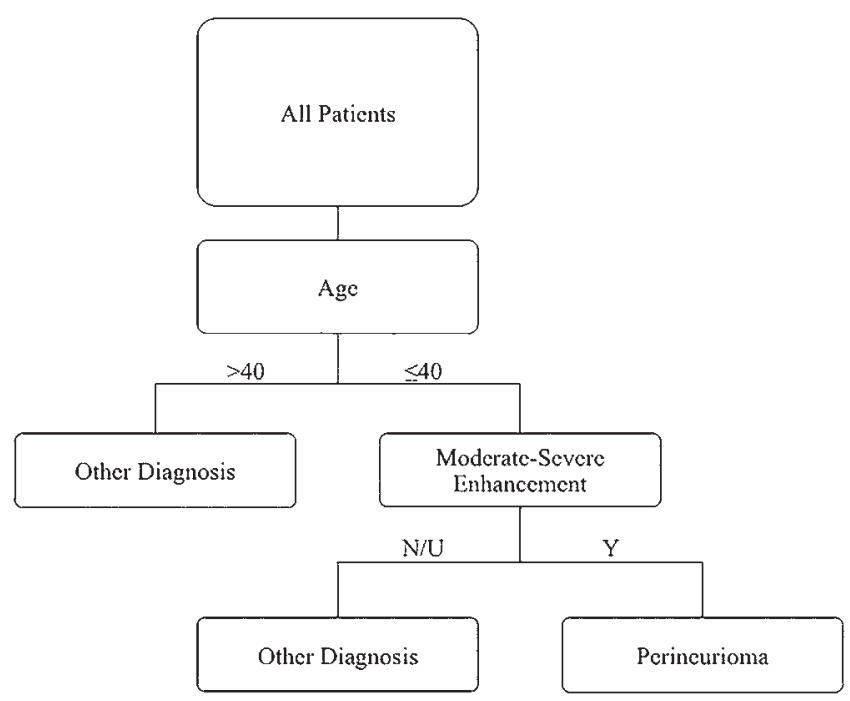

FIG. 4. Decision tree generated using both the $\mathrm{C} 5.0$ and $\mathrm{rPart}$ statistical packages in $\mathrm{R} . \mathrm{N}=$ no; $\mathrm{U}=$ unknown; $\mathrm{Y}=$ yes. cant predictors; increasing age and clinical history of cancer as negative predictors and moderate to severe contrast enhancement and homogeneous contrast enhancement as positive predictors of a diagnosis of perineurioma.

\section{Comparison of Methods for Predicting Perineurioma Diagnosis}

A total of 32 patients in the total cohort satisfied all 8 of the Perineurioma Diagnostic Criteria. All 32 (100\%) patients meeting the Perineurioma Diagnostic Criteria were diagnosed with a perineurioma.

Among patients in the cohort, 17 did not undergo postcontrast imaging, so we then applied the Perineurioma Diagnostic Criteria without those criteria related to postcontrast imaging (that is, we excluded criteria 4 [moderate to severe contrast enhancement] and 5 [homogeneous contrast enhancement]). A total of 47 patients satisfied these criteria; 40 of the $47(85 \%)$ patients were diagnosed with a perineurioma. The actual diagnoses for the 7 false positives were arteriovenous malformation, glomangiomyoma, inflammatory pseudotumor, microvasculitis, onion bulb neuropathy, and chronic inflammatory demyelinating polyradiculopathy for 2 patients.

We next considered the factors that were significant in multivariate logistic regression analysis as a diagnostic set: 1) age $\leq 40$ years, 2) no history of cancer, 3) moderate to severe contrast enhancement, and 4) homogeneous contrast enhancement. A total of 36 patients satisfied all 4 criteria; 35 of the 36 (97\%) patients were diagnosed with a perineurioma. The actual diagnosis for the 1 false positive was sarcoidosis.

Finally, we generated decision trees in C5.0 and rPart. Both decision trees generated were identical (Fig. 4). Only 2 factors were incorporated into the decision trees: age and moderate to severe contrast enhancement. These decision trees predicted a diagnosis of perineurioma for 49 patients; 44 of the 49 (90\%) patients were diagnosed with a perineurioma. The actual diagnoses for the 5 false positives were onion bulb neuropathy, sarcoidosis, glomangiomyoma, microvasculitis, and chronic inflammatory demyelinating polyradiculopathy. 
TABLE 4. Comparisons of clinicoradiological methods for diagnosing perineurioma

\begin{tabular}{lccccc}
\hline \multicolumn{1}{c}{ Method } & Sensitivity & Specificity & PPV & NPV & $\begin{array}{c}\text { S1 } \\
\text { Score }\end{array}$ \\
\hline $\begin{array}{l}\text { Perineurioma Diagnos- } \\
\text { tic Criteria }\end{array}$ & 0.63 & 1.0 & 1.0 & 0.88 & 0.77 \\
$\begin{array}{l}\text { Perineurioma Diagnos- } \\
\text { tic Criteria w/0 } \\
\text { contrast }\end{array}$ & 0.78 & 0.95 & 0.85 & 0.93 & 0.81 \\
\hline $\begin{array}{l}\text { Logistic regression } \\
\text { significant factors }\end{array}$ & 0.69 & 0.99 & 0.97 & 0.90 & 0.81 \\
\hline C5.0 decision tree & 0.86 & 0.97 & 0.90 & 0.95 & 0.88 \\
\hline rPart decision tree & 0.86 & 0.97 & 0.90 & 0.95 & 0.88 \\
\hline
\end{tabular}

$\mathrm{NPV}=$ negative predictive value; $\mathrm{PPV}=$ positive predictive value .

The sensitivity, specificity, positive predictive value, negative predictive value, and F1 score were calculated for each method (Table 4). The highest sensitivity was observed for the decision trees generated in C5.0 and rPart (0.86), highest specificity for the Perineurioma Diagnostic Criteria (1.0), highest positive predictive value for the Perineurioma Diagnostic Criteria (1.0), highest negative predictive value for the decision trees generated in C5.0 and $\mathrm{rPart}(0.95)$, and highest F1 score also for the decision trees $(0.88)$.

\section{Performance of Radiologists' Gestalt}

Radiologist 1 gave 31 patients a grade of 5 on the perineurioma confidence scale (that is, definitely a perineurioma), and Radiologist 2 gave 21 patients a grade of 5. All patients graded a 5 on the perineurioma confidence scale by either radiologist were diagnosed with a perineurioma.

Radiologist 1 gave 122 patients a grade of 1 on the perineurioma confidence scale (that is, definitely not a perineurioma); 120 of the 122 (98\%) patients were diagnosed with something other than a perineurioma. Radiologist 2 gave 116 patients a grade of 1 on the perineurioma confidence scale; all 116 (100\%) patients were diagnosed with something other than a perineurioma.

\section{Discussion}

Perineuriomas account for approximately $1 \%$ of nerve sheath tumors but may be underrecognized due to a lack of familiarity by many clinicians. ${ }^{7-9,13}$ Controversy exists regarding optimal management of these lesions, mainly because of conflicting reports regarding their natural history. ${ }^{6,912}$ Regardless, optimal management begins with diagnosis. To this point, the standard has been fascicular biopsy to obtain a pathological tissue diagnosis. We present here several methods for diagnosing perineurioma using clinical and radiological factors that may obviate the need for tissue diagnosis in certain circumstances.

The main advantage of the current study is the large size of the cohort, with all patients having a biopsy-proven diagnosis. To our knowledge, this represents the largest cohort to date of targeted fascicular biopsies and the largest cohort of biopsy-proven intraneural perineuriomas. We found that several clinical and radiological features occurred more often in patients diagnosed with perineurioma than in the group diagnosed with something other than perineurioma. These factors included younger age; no pain at presentation; lack of a history of cancer; unifocal disease; and lesion characteristics of moderate to severe hyperintensity on T2-weighted imaging, moderate to severe contrast enhancement, homogeneous contrast enhancement, and fusiform shape.

Interrater reliability was high for all imaging features assessed based on the percentage agreement of the 2 radiologists, which exceeded the $80 \%$ threshold for all features assessed. The $\kappa$ coefficient suggested at least substantial agreement for all imaging features assessed except for enlargement of the involved nerve(s), which was fair. The percent agreement was $97 \%$ for enlargement of the involved nerve(s). The $\kappa$ coefficient was lower due to the fact that the overwhelming majority of involved nerves were enlarged, increasing the likelihood that the agreement occurred purely by chance. Using both statistics together, however, we believe that the data support the reproducibility of our findings by showing high interrater reliability for all imaging features that were assessed..$^{15}$

To obviate the need for tissue diagnosis, the alternative method of diagnosis must have a high specificity and positive predictive value. With a high specificity and positive predictive value, false positives are minimized and one can be confident in the diagnosis. The patient can then be managed confidently with a diagnosis of a perineurioma. Sensitivity and negative predictive value are less important in this circumstance. High sensitivity and negative predictive value minimize false negatives, but in this case, if the alternative method predicts a nonperineurioma diagnosis, then the patient would need a fascicular biopsy for tissue diagnosis. In this way, false negatives will be avoided by obtaining a tissue diagnosis.

We compared the following 5 clinicoradiological methods for diagnosing a perineurioma: Perineurioma Diagnostic Criteria, Perineurioma Diagnostic Criteria minus contrast-related criteria, the set of significant features from multivariate logistic regression analysis, the C5.0 decision tree, and the rPart decision tree. The method with the highest specificity and positive predictive value was the Perineurioma Diagnostic Criteria. Both the specificity and positive predictive value were $100 \%$. Although we believe that in real-world practice there are likely to be rare exceptions where all criteria are met and yet the diagnosis is something other than a perineurioma, we think that, based on our data, this will be the very rare exception. Correspondingly, we believe that when all of the Perineurioma Diagnostic Criteria are satisfied, the need for tissue diagnosis is obviated. The other methods presented in this study may be helpful in counseling patients on the likelihood of having a perineurioma. However, we believe that the gold standard for obviating the need for tissue diagnosis should be the Perineurioma Diagnostic Criteria, due to their extremely high specificity and positive predictive value.

The gestalt of both radiologists in this study, particularly when they were confident (perineurioma confidence scale 1 or 5 ), was highly accurate. This should not be surprising because one would predict that their gestalt would 
be largely based on the Perineurioma Diagnostic Criteria. When either radiologist was confident that the lesion was a perineurioma (perineurioma confidence scale 5), the diagnosis in all cases was, in fact, a perineurioma. This underscores the importance of having experienced radiologists review MR images focused on peripheral nerve pathology. One benefit of the current study is formalization of a set of criteria that all radiologists ought to be able to apply, so that even those radiologists less experienced with perineuriomas should be able to identify them with high specificity.

It is important to note that many of the factors that were found to be important rely on the availability of postcontrast images. This fact underscores the importance of using contrast when evaluating peripheral nerve lesions. When excluding the contrast-related factors, the Perineurioma Diagnostic Criteria dropped to a positive predictive value of $85 \%$ (from $100 \%$ ). When evaluating for a perineurioma, the evaluation should be considered incomplete if postcontrast images are not available. Postcontrast images should be obtained prior to determining whether to proceed with fascicular biopsy for tissue diagnosis.

Many questions remain to be answered, including the neurological and radiological natural history of perineuriomas and the optimal management of perineuriomas. With regard to the natural history, the optimal radiological follow-up is yet to be determined. Management options may include resection, resection with nerve graft repair, nerve transfer, tendon transfer, or conservative management. When, if ever, each of these management options should be used requires further investigation. Based on the current study, we believe that it would be acceptable and appropriate to include those patients without tissue diagnosis but who satisfy the Perineurioma Diagnostic Criteria in future perineurioma cohorts for further study.

\section{Conclusions}

This study identifies clinical and radiological features that are associated with a diagnosis of intraneural perineurioma. To reiterate, the Perineurioma Diagnostic Criteria are as follows: 1) no cancer history, 2) unifocal disease, 3) moderate to severe hyperintensity on T2-weighted imaging, 4) moderate to severe contrast enhancement, 5) homogeneous contrast enhancement, 6) fusiform shape, 7) enlargement of the involved nerves, and 8) age $\leq 40$ years. Use of the Perineurioma Diagnostic Criteria obviates the need for tissue diagnosis when all of the criteria are satisfied.

\section{References}

1. Ariza A, Bilbao JM, Rosai J: Immunohistochemical detection of epithelial membrane antigen in normal perineurial cells and perineurioma. Am J Surg Pathol 12:678-683, 1988

2. Boyanton BL Jr, Jones JK, Shenaq SM, Hicks MJ, Bhattacharjee MB: Intraneural perineurioma: a systematic review with illustrative cases. Arch Pathol Lab Med 131:13821392, 2007

3. Chung JH, Jeong SH, Dhong ES, Han SK: Surgical removal of intraneural perineurioma arising in the brachial plexus using an interfascicular dissection technique. Arch Plast Surg 41:296-299, 2014

4. Emory TS, Scheithauer BW, Hirose T, Wood M, Onofrio BM, Jenkins RB: Intraneural perineurioma. A clonal neoplasm associated with abnormalities of chromosome 22. Am J Clin Pathol 103:696-704, 1995

5. Gisev N, Bell JS, Chen TF: Interrater agreement and interrater reliability: key concepts, approaches, and applications. Res Social Adm Pharm 9:330-338, 2013

6. Gruen JP, Mitchell W, Kline DG: Resection and graft repair for localized hypertrophic neuropathy. Neurosurgery 43:7883, 1998

7. Lee HY, Manasseh RG, Edis RH, Page R, Keith-Rokosh J, Walsh P, et al: Intraneural perineurioma. J Clin Neurosci 16:1633-1636, 2009

8. Macarenco RS, Ellinger F, Oliveira AM: Perineurioma: a distinctive and underrecognized peripheral nerve sheath neoplasm. Arch Pathol Lab Med 131:625-636, 2007

9. Mauermann ML, Amrami KK, Kuntz NL, Spinner RJ, Dyck PJ, Bosch EP, et al: Longitudinal study of intraneural perineurioma-a benign, focal hypertrophic neuropathy of youth. Brain 132:2265-2276, 2009

10. McHugh ML: Interrater reliability: the kappa statistic. Biochem Med (Zagreb) 22:276-282, 2012

11. McMillan HJ, Torres C, Michaud J, Ying Y, Boyd KU, Bourque PR: Diagnosis and outcome of childhood perineurioma. Childs Nerv Syst 32:1555-1560, 2016

12. Østergaard JR, Smith T, Stausbøl-Grøn B: Intraneural perineurioma of the sciatic nerve in early childhood. Pediatr Neurol 41:68-70, 2009

13. Restrepo CE, Amrami KK, Howe BM, Dyck PJ, Mauermann ML, Spinner RJ: The almost-invisible perineurioma. Neurosurg Focus 39(3):E13, 2015

14. Scheller C, Richter HP, Scheuerle A, Kretschmer T, König $\mathrm{RW}$, Antoniadis G: Intraneural perineuriomas; a rare entity. Clinical, surgical and neuropathological details in the management of these lesions. Zentralbl Neurochir 69:134-138, 2008

15. Steinijans VW, Diletti E, Bömches B, Greis C, Solleder P: Interobserver agreement: Cohen's kappa coefficient does not necessarily reflect the percentage of patients with congruent classifications. Int J Clin Pharmacol Ther 35:93-95, 1997

\section{Disclosures}

The authors report no conflict of interest concerning the materials or methods used in this study or the findings specified in this paper.

\section{Author Contributions}

Conception and design: Wilson, Spinner, Amrami. Acquisition of data: Wilson, Howe, Stewart, Amrami. Analysis and interpretation of data: Wilson, Howe, Spinner, Amrami. Drafting the article: all authors. Critically revising the article: all authors. Reviewed submitted version of manuscript: Wilson, Howe, Stewart, Spinner. Approved the final version of the manuscript on behalf of all authors: Wilson. Statistical analysis: Wilson. Study supervision: Spinner, Amrami.

\section{Correspondence}

Thomas J. Wilson, Department of Neurosurgery, Stanford University, 300 Pasteur Dr., R293, Stanford, CA 94305. email: wilsontj@ stanford.edu. 